\title{
Synthesis of New 2-Aminocarbohydrate-1,4-Naphthoquinone Derivatives Promoted by Ultrasonic Irradiation
}

\author{
Caroline F. J. Franco, ${ }^{a}$ Alessandro K. Jordão, ${ }^{a}$ Vitor F. Ferreira, ${ }^{a}$ Angelo C. Pinto, ${ }^{c}$ \\ Maria C. B. V. de Souza, ${ }^{a}$ Jackson A. L. C. Resende ${ }^{b}$ and Anna C. Cunha*,a \\ ${ }^{a}$ Departamento de Química Orgânica, Instituto de Química and ${ }^{b}$ Departamento de Química \\ Inorgânica, Laboratório Regional de Difração de Raios X (LDRX), Outeiro de São João Batista, \\ Universidade Federal Fluminense, 24020-141 Niterói-RJ, Brazil \\ 'Departamento de Química Orgânica, Instituto de Química, Universidade Federal do Rio de Janeiro, \\ Cidade Universitária, 21941-590 Rio de Janeiro-RJ, Brazil
}

\begin{abstract}
Neste trabalho nós descrevemos o uso do ultrassom na síntese de novas naftoquinonas, $6 \mathbf{a}-\mathbf{f}$ e 7a-c, contendo na posição 2 do anel quinonoídico substituintes do tipo aminocarboidratos. Estas substâncias foram preparadas, em condições brandas, através da reação da 1,4-naftoquinona (8a) ou do metoxi-lapachol (8b) com diferentes aminocarboidratos 9a-d. As estruturas das substâncias foram confirmadas através das técnicas de $\mathrm{RMN}$ de ${ }^{1} \mathrm{H}$ e de ${ }^{13} \mathrm{C}$-APT, uni- e bi-dimensionais (COSY${ }^{1} \mathrm{H}$ vs. ${ }^{1} \mathrm{H}$ e HETCOR ${ }^{1} J_{\mathrm{CH}}$ ), e por espectrometria de massas de alta resolução com ionização por electrospray (ESI MS).
\end{abstract}

In this report we describe the ultrasound-accelerated synthesis of new naphthoquinone derivatives 6a-f and 7a-c, which possess an aminocarbohydrate chain at the $\mathrm{C}-2$ position of the quinone ring. This novel type of 1,4-naphthoquinone derivative has been synthesized under mild conditions by the reaction of 1,4-naphthoquinone (8a) or methoxylapachol $(\mathbf{8 b})$ with different aminocarbohydrates $\mathbf{9 a - d}$. Characterization of all substances was confirmed by oneand two-dimensional nuclear magnetic resonance (NMR) techniques $\left({ }^{1} \mathrm{H},{ }^{13} \mathrm{C}\right.$-APT, cosy- ${ }^{1} \mathrm{H}$ vs. ${ }^{1} \mathrm{H}$ and HETCOR ${ }^{1} J_{\mathrm{CH}}$ ) and by high-resolution electrospray ionization mass spectrometry (HR ESI MS).

Keywords: naphthoquinones, aminocarbohydrates, sonochemistry

\section{Introduction}

The naphthoquinones are important natural substances that are widely distributed in different families of plants, fungi and some animals; ${ }^{1}$ many of them play integral roles in vital biochemical processes. For example, vitamins $\mathrm{K}_{1}$ and $\mathrm{K}_{2}$ are natural naphthoquinones that are responsible for the human blood coagulation mechanism. ${ }^{2}$ Due to this involvement in metabolism, this class of substances has been extensively studied concerning their various biological properties. Many of them have ceased to be mere curiosities and have become very useful drugs for mankind. ${ }^{3}$

Recent reports in the literature ${ }^{2,4-8}$ point out that their mechanisms of action normally involve the generation of active oxygen species (e.g., superoxide radical anions,

\footnotetext{
*e-mail: annac@vm.uff.br
}

hydrogen peroxide and hydroxyl radicals) by redox cycling, intercalation in the DNA double helix or alkylation of biomolecules. In all of these cases, the biological activity requires bioreduction of the quinone nucleus as the first activating step. ${ }^{6,8}$ As the bioreduction of the quinones is influenced by their redox properties, the electron-accepting capacity of these compounds can be modified by adding electron-attracting or donating substituents to the quinone system. ${ }^{4,5-12}$ The introduction of vicinal oxidizable groups, such as amino substituents, onto the quinone nucleus can exert influence on its redox properties, inducing oxidative stress in cells, and alkylation of cellular macromolecules, including lipids, proteins and DNA. ${ }^{2,4}$ Indeed, the literature to date clearly shows that aminoquinone frameworks are present in several important bioactive compounds that exhibit antitumor ${ }^{3} \mathbf{1}$, antimalarial ${ }^{13} \mathbf{2}$, antithrombotic ${ }^{14} \mathbf{3}$, antifungal $^{9} \mathbf{4}$ and molluscicidal ${ }^{15}$ activities $\mathbf{5}$ (Figure 1). 

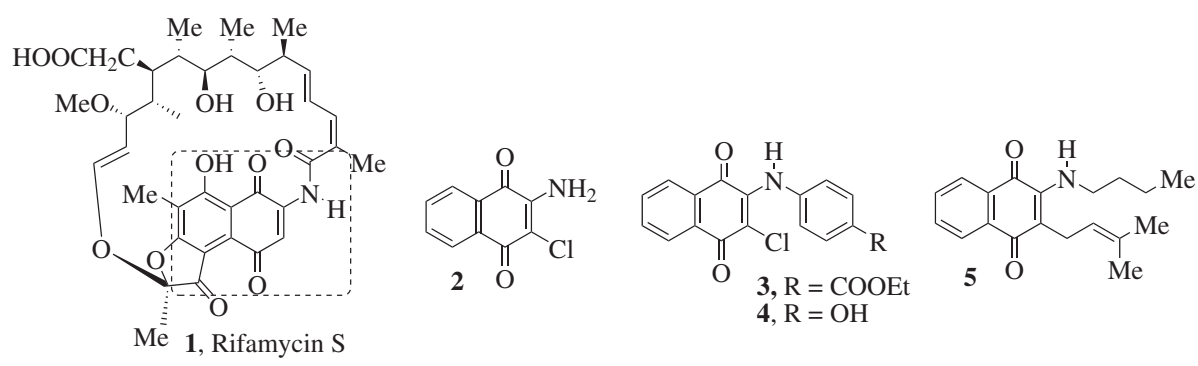

Figure 1. Some examples of bioactive products with the aminonaphthoquinone moiety.

Several methods for the conversion of quinones into their amino derivatives have been reported in the literature: a) reduction of nitroso-quinone compounds ${ }^{16}$ with sodium dithionate; b) reaction of hydrazoic acid with quinones, ${ }^{17,18} \mathrm{c}$ ) nucleophilic substitution reactions of 2-methoxy-quinone $e^{4,10,15,19,20}$ or halo-derivatives ${ }^{21-23}$ with amines; d) direct 1,4-type addition of amines to the quinones with ${ }^{24}$ or without ${ }^{25,26}$ Lewis acid catalysis (e.g., $\mathrm{CeCl}_{3} \cdot \mathrm{H}_{2} \mathrm{O}$ ); e) Lewis acid catalyzed addition (e.g., $\mathrm{CeCl}_{3}, \mathrm{CAN}, \mathrm{FeCl}_{3}$ and $\mathrm{I}_{2}$ ) under ultrasonic irradiation; ${ }^{27}$ f) nuclear amination of $p$-hydroquinones with primary aromatic amines catalyzed by fungal laccases; $;^{28}$ and $g$ ) Michael-type addition of 2,3-dichloronaphthoquinone with potassium cyanate..$^{29}$

Sugar-containing quinones ${ }^{30,31}(O$-glycoside derivatives) or $N$-heterocyclic moiety ${ }^{32}$ have been found in many compounds possessing significant antitumor activity.

Although these methods previously described have been used for the synthesis of aminoquinones, there is a lack of synthetic studies for the preparation of sugaraminonaphthoquinones. Additionally, the development of a versatile method for their preparation in mild conditions is of great importance since it is possible that these new substances can constitute a new class of bioactive naphthoquinone derivatives.

As part of an ongoing research program on the synthesis of new biologically active naphthoquinones and on the basis of our experience in the field of the use of readily available carbohydrates ${ }^{33,34}$ in organic reactions, herein we report the ultrasound-accelerated synthesis of new naphthoquinone derivatives 6a-d and 7a-c (Schemes 1 and 2) possessing an aminocarbohydrate chain at C-2 position of the quinone moiety.

\section{Results and Discussion}

Methyl 5-amino-5-deoxy-1,2- $O$-isopropylidene$\beta$-D-ribofuranoside 9a, 6-amino-1,2:3,4-di- $O$ isopropylidene- $\alpha$-D-galactopyranose $\mathbf{9 b}$ and 5 -amino5-deoxy-1,2-O-isopropylidene- $\alpha$-D-xilofuranose 9c were prepared from their corresponding commercially reagents D-ribose, D-xylose and D-galactose by using known methods of carbohydrate protection and derivatization. ${ }^{35-37}$ The ultrasound-accelerated 1,4-addition of aminocarbohydrates 9a-c to 1,4-naphthoquinone (8a) was first carried out in the presence of iodine at room temperature, following the procedure described in the literature ${ }^{27}$ (Scheme 1, method A). The novel aminonaphthoquinones $\mathbf{6 a - c}$ were purified by flash column chromatography using 50\% $n$-hexane/ ethyl acetate as eluent. The yields of these reactions are listed in Table 1. The reaction of D-glucosamine hydrochloride 9d using method A failed to produce the compound $\mathbf{6 d}$. Thus, the reaction under ultrasound irradiation was also investigated in the absence of iodine (Scheme 1, method B). This method led to better yields of the aminonaphthoquinones $\mathbf{6 a - c}$, as shown in the Table 1. In this case, the 1,4-addition reaction between naphthoquinone 8a and D-glucosamine hydrochloride 9d resulted in the compound $\mathbf{6 d}$, as a mixture of two diastereoisomers, $\alpha: \beta(1: 1)$ in $40 \%$ yield. The treatment of this mixture with acetic anhydride in pyridine in excess produced the desired tetraacetate derivative in $60 \%$ yield as a mixture of anomers $(3: 1)$, being $6 \mathrm{e}$ the major isomer. The yields of the quinones $\mathbf{6 a - d}$ were dependent on the structure of the aminocarbohydrates 9a-d in question. The reactions worked very well with primary amines 9a-c, affording the corresponding coupling products in good yields, while more hindered amine, e.g., 9d, led to the product $\mathbf{6 d}$ in moderate yield.

The characterization of the aminonaphthoquinone structures 6a-c and 6e was performed by using one- and two-dimensional NMR techniques and by HR ESI MS. The preferential conformations of the carbohydrates were assigned based upon the proton coupling constants $(J)$ of furan or pyran ring, and by chemical shift comparison with previous data reported in the literature for analogous carbohydrates. ${ }^{38-41}$

The ${ }^{1} \mathrm{H}$ NMR spectrum of compound $\mathbf{6 a}$ showed the anomeric proton $\left(\mathrm{H}-1^{\prime}\right)$ as a singlet at $\delta 4.98$. The protons $\mathrm{H}-2$ ' and $\mathrm{H}-3$ ' appeared as doublets at $\delta 4.59$ 


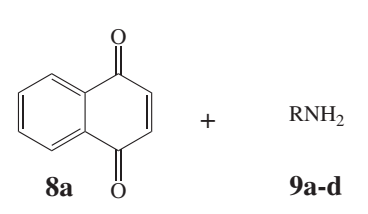

a) Method $\mathrm{A}: \mathrm{I}_{2} / \mathrm{MeOH}$ anhydrous / r.t.

b) Method B: $\mathrm{MeOH} /$ r.t.

c) acetic anhydride / r.t. a) or b)

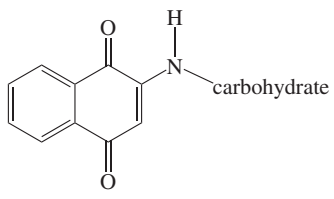

6a-c
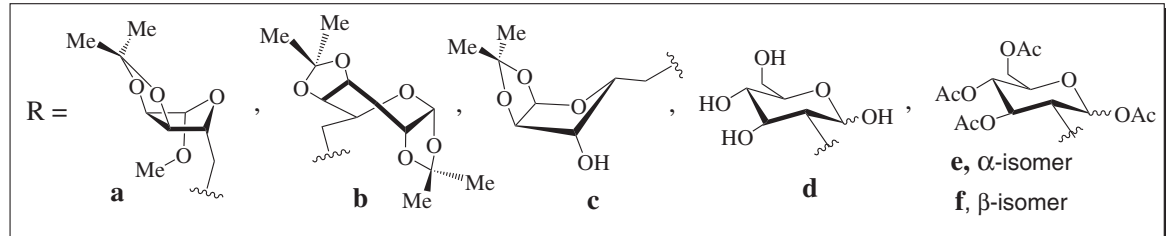

Scheme 1. Synthesis of novel 2-aminonaphthoquinones 6a-f.

Table 1. Aminonaphthoquinones 6a-d and 7a-c reaction yields

\begin{tabular}{lcccc}
\hline Quinones & carbohydrates & Products & \multicolumn{2}{c}{ Methods $^{\text {a }}$} \\
& & & A Yield \% & B Yield \% \\
\hline 8a & 9a & 6a & 63 & 73 \\
8a & 9b & 6b & 51 & 70 \\
8a & 9c & 6c & 68 & 75 \\
8a & 9d & 6d & - & 40 \\
8b & 9a & $\mathbf{7 a}$ & - & 20 \\
8b & 9b & $\mathbf{7 b}$ & - & 27 \\
$\mathbf{8 b}$ & 9c & $\mathbf{7 c}$ & - & 37 \\
\hline
\end{tabular}

${ }^{\mathrm{a}}$ Method A: $\mathrm{I}_{2} / \mathrm{MeOH}$ anhydrous / r.t.; Method B: $\mathrm{MeOH} /$ r.t..

$\left(J_{\mathrm{H}-2^{\prime}, \mathrm{H}-3^{3}}=6.1 \mathrm{~Hz}\right)$ and $\delta 4.57\left(J_{\mathrm{H}-3^{\prime}, \mathrm{H}-2^{\prime}}=6.1 \mathrm{~Hz}\right)$, respectively. The protons $\mathrm{H}-4$ ' and $\mathrm{H}-5$ ' were identified as multiplets at $\delta$ 4.41-4.45 and 3.16-3.29, respectively. The absence of vicinal coupling indicates a trans relationship between protons $\mathrm{H}-1^{\prime}$ ' and $\mathrm{H}-2^{\prime}$ ' and the $\beta$-anomeric configuration for the carbohydrate. ${ }^{41}$ The envelope conformations ${ }^{\circ} E$ and ${ }^{4} E$ for compounds $6 \mathbf{a}$ and $\mathbf{6 c}$, respectively, were established on the basis of X-ray crystallographic data of 5-modified analogous of methyl $2,3-O$-isopropylidene- $\beta$-ribofuranoside ${ }^{42}$ and 5-deoxy$1,2-O$-isopropylidene- $\alpha$-D-xylofuranose. ${ }^{43}$

The twist-boat conformation of the D-galactose ring in 6b was confirmed by the $1 \mathrm{H}-1 \mathrm{H}$ vicinal coupling constants values $J_{\mathrm{H}-1^{\prime}, \mathrm{H}-2^{\prime}}, J_{\mathrm{H}-2^{2}, \mathrm{H}-3^{3}}, J_{\mathrm{H}-3^{\prime}, \mathrm{H}-4^{2}}$ and $J_{\mathrm{H}-4^{\prime}, \mathrm{H}-5^{\prime}},(5.0,2.4,8.0$ and $1.5 \mathrm{~Hz}$, respectively) of the ring protons. ${ }^{38}$

The $\alpha: \beta$ ratio of D-glucosamine derivatives $6 \mathbf{e}$ and 6f was determined by the areas of the H-1' peaks of the respective anomers in the ${ }^{1} \mathrm{H}$ NMR spectra. The $\alpha$-configuration at position C-1' of the major isomer $\mathbf{6 e}$ is supported by the small coupling constant between $\mathrm{H}-1^{\prime}$ and $\mathrm{H}-2^{\prime}\left(\mathrm{d}, J_{\mathrm{H}-\mathrm{I}^{\prime}, \mathrm{H}-\mathrm{2}^{\prime}}=4.0 \mathrm{~Hz}\right)$. The structures of the two possible chair conformations $\left({ }^{4} \mathrm{C}-1\right.$ and $\left.{ }^{1} \mathrm{C}-4\right)$ of the $\alpha$-pyranose form are shown in Figure 2. The large vicinal coupling constants for the protons ${ }^{3} \mathrm{~J}_{\mathrm{H}-2, \mathrm{H}-3}$, and ${ }^{3} J_{\mathrm{H}-3^{\prime}, \mathrm{H}-4^{\prime}}(10.0$ and $9.5 \mathrm{~Hz}$, respectively) are in agreement with trans-diaxial arrangements for the protons $\mathrm{H} 2$ '- $-\mathrm{H} 3$ ' and $\mathrm{H}^{\prime}$ '- $\mathrm{H} 4$ ', suggesting a ${ }^{4} \mathrm{C}-1$ conformation for the pyran ring.

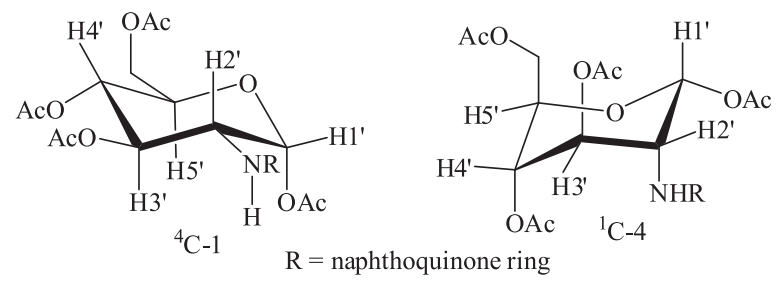

Figure 2. Two possible conformations of pyranose ring of $\mathbf{6 e}$.

The use of 2-methoxy-3-(3-methyl-2-butenyl)-1,4naphthoquinone (8b) (2-methoxylapachol) in ultrasoundaccelerated reaction (Scheme 2) with aminocarbohydrates 9a-c was also studied. The novel sugar-lapachol derivatives $7 \mathbf{a}-\mathbf{c}$ were prepared in moderated yields by nucleophilic substitution reaction of $\mathbf{8 b}$ with amines $9 \mathbf{9}-\mathbf{c}$ (Table 1).

For comparison purposes, the reactions of amine $9 \mathbf{a}$ with naphthoquinone 8a or methoxylapachol $\mathbf{8 b}$ were carried out under conventional heating. In both cases, no conversion into products was observed after a $2 \mathrm{~h}$ reaction, indicating that the ultrasound irradiation is essential for the activation and performing of these coupling reactions. The effects of sonication on reactions can be related to high temperature and pressure resulting from acoustic cavitation. ${ }^{44}$

The structures proposed for compounds $\mathbf{7 a}, \mathbf{7 b}$ and $\mathbf{7 c}$ are supported by their HR ESI MS and NMR spectroscopic data $\left({ }^{1} \mathrm{H}\right.$, APT, HETCOR ${ }^{1} J_{\mathrm{CH}}$ and $\left.{ }^{\mathrm{n}} J_{\mathrm{CH}}, \mathrm{n}=2,3\right)$. 

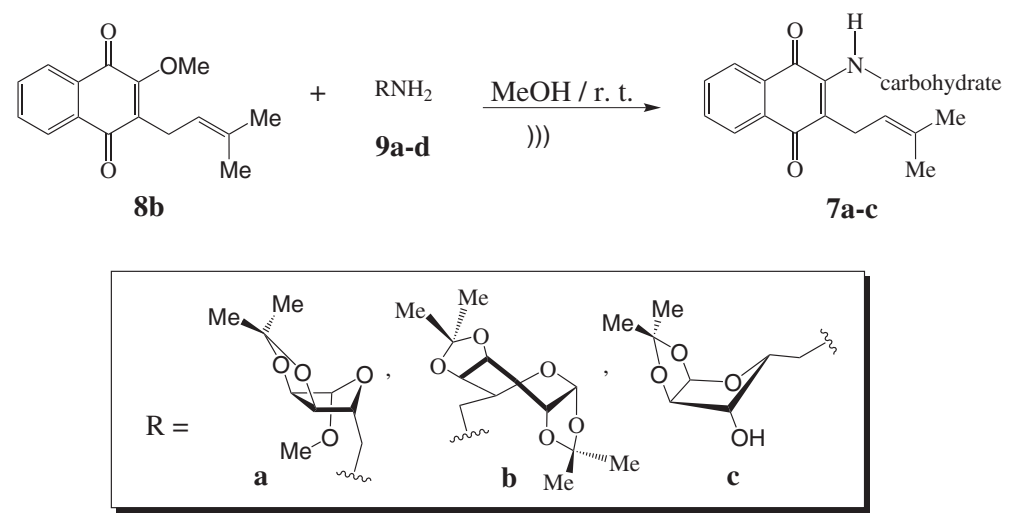

Scheme 2. Synthesis of 1,4-naphthoquinone derivatives 7a-c.

\section{Experimental}

Chemical reagents and all solvents used in this study were purchased from Merck AG (Darmstadt, Germany) and VETEC LTDA. The synthesis of new aminonaphthoquinones 6a-d and 7a-c was performed in an ultrasonic bath $(40 \mathrm{KHz})$ model USC 1400, ultrasonic cleaner, UNIQUE. Melting points were determined with a Fisher-Johns instrument and are uncorrected. Infrared (IR) spectra were recorded on Perkin-Elmer FT-IR model 1600 spectrophotometer, in $\mathrm{KBr}$ pellets. NMR spectra, unless otherwise stated, were obtained in deuterated $\mathrm{CDCl}_{3}$ using a Varian Unity Plus $300 \mathrm{MHz}$ spectrometer. Chemical shifts $(\delta)$ are expressed in ppm and the coupling constants $(J)$ in Hertz. High-resolution electrospray ionization mass spectrometry (HR ESI MS) was performed in positive ion mode on a Waters-Micromass Q-Tof Micro instrument. The progress of all reactions was monitored by TLC performed on $2.0 \mathrm{~cm} \times 6.0 \mathrm{~cm}$ aluminum sheets precoated with silica gel 60 (HF-254, E. Merck) to a thickness of $0.25 \mathrm{~mm}$. The developed chromatograms were viewed under ultraviolet light at $254 \mathrm{~nm}$. Merck silica gel (60-200 mesh) was used for column chromatography.

\section{Synthesis}

General procedure for the preparation of the novel aminonaphthoquinones $\mathbf{6 a - d}$ and $\mathbf{7 a - c}$

A solution of naphthoquinone $8 \mathbf{a}$ or $8 \mathbf{b}(0.5 \mathrm{mmol})$ and aminocarbohydrate $9(0.5 \mathrm{mmol})$ in methanol $(\mathrm{MeOH})(2$ $\mathrm{mL}$ ) was sonicated for $2 \mathrm{~h}$ at r.t.. The solvent was removed under reduced pressure. The residue was purified by flash column chromatography (gradient elution, 10-30\% AcOEt in hexane) to afford the desired compounds 6a-c and 7a-c.

2-(Methyl-5'-deoxy-2',3'-O-isopropylidene- $\beta$-Dribofuranosid-5'-yl)-amino-1,4- naphthoquinone, $6 \mathbf{a}$

The reaction between naphthoquinone $\mathbf{8 a}(158 \mathrm{mg}$,
$1 \mathrm{mmol})$ and aminocarbohydrate $9 \mathrm{a}(101 \mathrm{mg}, 0.5 \mathrm{mmol})$ yielded $\mathbf{6 a}$ (74 $\mathrm{mg}, 0.2 \mathrm{mmol}, 73 \%$ ) as a brown solid: $\mathrm{mp}$ 140-142 ${ }^{\circ} \mathrm{C}$; IR (KBr) $v_{\max } / \mathrm{cm}^{-1} 3244(\mathrm{~N}-\mathrm{H}) ; 1683$ and $1601(\mathrm{C}=\mathrm{O}), 1567(\mathrm{C}=\mathrm{C}) ;{ }^{1} \mathrm{H}$ NMR $\left(300 \mathrm{MHz}, \mathrm{CDCl}_{3}\right)$ $\delta 1.25\left(\mathrm{~s}, 3 \mathrm{H}, \mathrm{C}\left(\mathrm{CH}_{3}\right)_{2}\right), 1.42\left(\mathrm{~s}, 3 \mathrm{H}, \mathrm{C}\left(\mathrm{CH}_{3}\right)_{2}\right), 3.16-3.29$ (m, 2H, H-5' and H-5"), 3.34 (s, 3H, $\left.\mathrm{OCH}_{3}\right), 4.41-4.45$ (m, 1H, H-4'), 4.57 (d, 1H, J 6.1, H-3'), 4.59 (d, 1H, J 6.1 Hz, H-2'), 4.98 (s, 1H, H-1'), 5.67 (s, 1H, H-3), 6.40 (dd, $1 \mathrm{H}, J 4.4 \mathrm{~Hz}, \mathrm{~N}-\mathrm{H}), 7.54$ (td, $J 7.5$ and $1.5 \mathrm{~Hz}, \mathrm{H}-7), 7.65$ (td, $J 7.5$ and $1.5 \mathrm{~Hz}, \mathrm{H}-6$ ), 7.98 (dd, $J 7.9$ and $1.5 \mathrm{~Hz}, \mathrm{H}-8$ ), 8.03 (dd, J 7.5 and $1.5 \mathrm{~Hz}, \mathrm{H}-5) ;{ }^{13} \mathrm{C}$ NMR $(75.0 \mathrm{MHz}$,

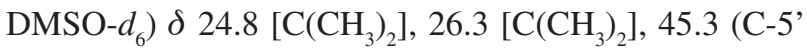
and C-5"), $55.3\left(\mathrm{OCH}_{3}\right), 82.1$ (C-3'), 84.3 (C-4'), 85.2 (C-2'), 101.3 (C-3), 109.5 (C-1'), 112.7 [C( $\left.\left.\mathrm{CH}_{3}\right)_{2}\right], 126.1$ (C-5), 126.2 (C-8), 130.4 (C-8a), 132.0 (C-7), 133.4 (C-4a), 134.6 (C-6), 148.0 (C-2), 182.9 (C-1), 198.9 (C-4); HRMS (ESI) $\left[\mathrm{M}^{+} \mathrm{Na}\right]^{+}$Found: 382.1256. Calc. for $\mathrm{C}_{19} \mathrm{H}_{21} \mathrm{NO}_{6} \mathrm{Na}$ : 382.1261 .

2-(6'-Deoxy-1',2':3', 4'-di-O-isopropylidene-Dgalactopyranos-6'-yl)-amino-1,4- naphthoquinone, $\mathbf{6 b}$

The reaction of naphthoquinone $\mathbf{8 a}(158 \mathrm{mg}, 1 \mathrm{mmol})$ and aminocarbohydrate $9 \mathbf{b}(128 \mathrm{mg}, 0.5 \mathrm{mmol})$ formed $\mathbf{6 b}$ (90 mg, $0.22 \mathrm{mmol}, 70 \%$ ) as a brown solid: $\mathrm{mp} 60-62^{\circ} \mathrm{C}$; IR $v_{\max } / \mathrm{cm}^{-1} 1609$ and $1678(\mathrm{C}=\mathrm{O}), 1572(\mathrm{C}=\mathrm{C}), 3379(\mathrm{~N}-\mathrm{H})$; ${ }^{1} \mathrm{H}$ NMR $\left(300 \mathrm{MHz}\right.$, DMSO- $\left.d_{6}\right) \delta 1.35\left(\mathrm{~s}, 3 \mathrm{H}, \mathrm{C}\left(\mathrm{CH}_{3}\right)_{2}\right)$, $1.42\left(\mathrm{~s}, 3 \mathrm{H}, \mathrm{C}\left(\mathrm{CH}_{3}\right)_{2}\right), 1.45\left(\mathrm{~s}, 3 \mathrm{H}, \mathrm{C}\left(\mathrm{CH}_{3}\right)_{2}\right), 1.52(\mathrm{~s}, 3 \mathrm{H}$, $\left.\mathrm{C}\left(\mathrm{CH}_{3}\right)_{2}\right), 3.40-3.51$ (m, 2H, H-6' and H-6"), 4.01-4.10 (m, $1 \mathrm{H}, \mathrm{H}-5$ '), 4.25 (dd, $1 \mathrm{H}, J 8.0$ and $\left.1.5 \mathrm{~Hz}, \mathrm{H}-4^{\prime}\right), 4.34$ (dd, $1 \mathrm{H}, J 5.0$ and $\left.2.4 \mathrm{~Hz}, \mathrm{H}-2^{\prime}\right), 4.73(\mathrm{dd}, 1 \mathrm{H}, J 8.0$ and 2.4 Hz, H-3'), 5.56 (d, 1H, J 5.0 Hz, H-1'), 5.80 (s, 1H, H-3), $7.58(\mathrm{dd}, 1 \mathrm{H}, J 6.2 \mathrm{~Hz}, \mathrm{~N}-\mathrm{H}), 7.61$ (td, $1 \mathrm{H}, J 7.5$ and 1.5 Hz, H-7), 7.72 (td, 1H, J 7.5 and 1.5 Hz, H-6), 8.04 (dd, $1 \mathrm{H}, J 7.5$ and $1.5 \mathrm{~Hz}, \mathrm{H}-8), 8.10$ (dd, $1 \mathrm{H}, J 7.5$ and $1.5 \mathrm{~Hz}$, $\mathrm{H}-5) ;{ }^{13} \mathrm{C}$ NMR $\left(75.0 \mathrm{MHz}\right.$, DMSO- $\left.d_{6}\right) \delta 24.5\left[\mathrm{C}\left(\mathrm{CH}_{3}\right)_{2}\right]$, $25.0\left[\mathrm{C}\left(\mathrm{CH}_{3}\right)_{2}\right], 25.9\left[\mathrm{C}\left(\mathrm{CH}_{3}\right)_{2}\right], 26.2\left[\mathrm{C}\left(\mathrm{CH}_{3}\right)_{2}\right], 42.5(\mathrm{C}-$ 6' and C-6”), 64.7 (C-5'), 70.1 (C-2'), 70.5 (C-3'), 71.0 
(C-4'), 95.9 (C-1'), 100.2 (C-3), 108.3 [C( $\left.\left(\mathrm{CH}_{3}\right)_{2}\right], 108.9$ $\left[\mathrm{C}\left(\mathrm{CH}_{3}\right)_{2}\right], 125.6(\mathrm{C}-5), 126.2(\mathrm{C}-8), 130.5$ (C-8a), 132.6 (C-7), 133.2 (C-4a), 135.2 (C-6), 148.7 (C-2), 181.7 (C-1 or C-4), 181.8 (C-1 or C-4); HRMS (ESI) [M+Na] ${ }^{+}$Found: 438.1541. Calc. for $\mathrm{C}_{22} \mathrm{H}_{25} \mathrm{NO}_{7} \mathrm{Na}$ : 438.1523 .

2-(5'-Deoxy-1',2'-O-isopropylidene-D-xylofuranos-5'yl)-amino-1,4- naphthoquinone, $\mathbf{6 c}$

The reaction of naphthoquinone $\mathbf{8 a}(158 \mathrm{mg}, 1 \mathrm{mmol})$ and aminocarbohydrate $9 \mathrm{c}(95 \mathrm{mg}, 0.5 \mathrm{mmol})$ led to formation of $\mathbf{6 c}$ ( $72 \mathrm{mg}, 0.2 \mathrm{mmol}, 75 \%$ ) as a brown solid: $\mathrm{mp} 160-163{ }^{\circ} \mathrm{C}$; IR $v_{\max } / \mathrm{cm}^{-1} 1608$ and $1679(\mathrm{C}=\mathrm{O}), 1566$ $(\mathrm{C}=\mathrm{C}), 3390(\mathrm{~N}-\mathrm{H}) ;{ }^{1} \mathrm{H}$ NMR $\left(300 \mathrm{MHz}, \mathrm{DMSO}-d_{6}\right) \delta$ 1.33 (s, $\left.3 \mathrm{H}, \mathrm{C}\left(\mathrm{CH}_{3}\right)_{2}\right), 1.51$ (s, 3H, $\left.\mathrm{C}\left(\mathrm{CH}_{3}\right)_{2}\right), 3.43-3.61$ (m, 2H, H-5' and $\mathrm{H}-5$ "), 4.16 (dd, $1 \mathrm{H}, J 4.8$ and $2.9 \mathrm{~Hz}$, H-3'), 4.36-4.42 (m, 1H, H-4'), 4.56 (d, 1H, J 4.0 Hz, H-2'), 5.62 (d, $1 \mathrm{H}, J 4.0 \mathrm{~Hz}, \mathrm{OH}), 5.89$ (s, 1H, H-3), 5.97 (d, $1 \mathrm{H}, J 4.0 \mathrm{~Hz}, \mathrm{H}-1^{\prime}$ ), 7.60 (dd, 1H, J 6.0 and 6.2 $\mathrm{Hz}, \mathrm{N}-\mathrm{H}$ ), 7.85 (td, $1 \mathrm{H}, J 7.5$ and $1.5 \mathrm{~Hz}, \mathrm{H}-7), 7.95$ (td, $1 \mathrm{H}, J 7.5$ and $1.5 \mathrm{~Hz}, \mathrm{H}-6), 8.06$ (dd, $1 \mathrm{H}, J 7.5$ and 1.5 $\mathrm{Hz}, \mathrm{H}-8), 8.10$ (dd, $1 \mathrm{H}, J 7.5$ and $1.5 \mathrm{~Hz}, \mathrm{H}-5) ;{ }^{13} \mathrm{C}$ NMR $\left(75.0 \mathrm{MHz}, \mathrm{DMSO}-d_{6}\right) \delta 26.1\left[\mathrm{C}\left(\mathrm{CH}_{3}\right)_{2}\right], 26.7\left[\mathrm{C}\left(\mathrm{CH}_{3}\right)_{2}\right]$, 41.1 (C-5' and C-5'), 73.9 (C-3'), 77.8 (C-4'), 85.0 (C-2'), 99.7 (C-3), 104.4 (C-1'), 110.8 [C( $\left.\left.\mathrm{CH}_{3}\right)_{2}\right], 125.5(\mathrm{C}-5)$, 126.0 (C-8), 130.4 (C-8a), 132.4 (C-7), 133.0 (C-4a), 135.0 (C-6), 148.8 (C-2), 181.6 (C-1 or C-4), 181.7 (C-1 or $\mathrm{C}-4)$; HRMS (ESI) $\left[\mathrm{M}^{+} \mathrm{Na}\right]^{+}$Found: 368.1112. Calc. for $\mathrm{C}_{18} \mathrm{H}_{19} \mathrm{NO}_{6} \mathrm{Na}$ : 368.1104 .

2-(Methyl-5"'-deoxy-2",,3"'-O-isopropylidene- $\beta-D$ ribofuranosid-5"'-yl)-amino-3-(3'-methyl-buten-2'-yl)-1,4naphthoquinone, $7 a$

The reaction of naphthoquinone $\mathbf{8 b}$ (158 mg, $1 \mathrm{mmol})$ and aminocarbohydrate $9 \mathrm{a}(101 \mathrm{mg}, 0.5 \mathrm{mmol})$ formed 7a (43 mg, $0.1 \mathrm{mmol}, 20 \%$ ) as a brown oil; IR $v_{\max } / \mathrm{cm}^{-1}$ 1670 and $1732(\mathrm{C}=\mathrm{O}), 1600(\mathrm{C}=\mathrm{C}), 3343(\mathrm{~N}-\mathrm{H}) ;{ }^{1} \mathrm{H}$ NMR $\left(500 \mathrm{MHz}, \mathrm{DMSO}-d_{6}\right) \delta 1.35\left(\mathrm{~s}, 3 \mathrm{H}, \mathrm{C}\left(\mathrm{CH}_{3}\right)_{2}\right), 1.48(\mathrm{~s}, 3 \mathrm{H}$, $\left.\mathrm{C}\left(\mathrm{CH}_{3}\right)_{2}\right), 1.75$ (s, 3H, H-1'), 1.82 (s, 3H, H-5'), 3.35 (s, $\left.3 \mathrm{H}, \mathrm{OCH}_{3}\right), 3.37$ (d, $1 \mathrm{H}, J 6.0 \mathrm{~Hz}, \mathrm{H}-4$ '), $3.69-3.72(\mathrm{~m}, 2 \mathrm{H}$, H-5" and H-5"'), 4.42 (dd, $1 \mathrm{H}, J 7.0$ and $6.5 \mathrm{~Hz}, \mathrm{H}-4$ "), 4.68 (d, 1H, J 6.0 Hz, H-3"), 4.86 (d, 1H, J 6.0 Hz, H-2"), 5.10 (s, 1H, H-1"), 5.14 (t, 1H, J 6.0 Hz, H-3'), 6.63 (dd, $1 \mathrm{H}, J 6.5$ and $6.0 \mathrm{~Hz}, \mathrm{~N}-\mathrm{H}), 7.83$ (td, $1 \mathrm{H}, J 7.5$ and 1.5 Hz, H-7), 7.91 (td, 1H, J 7.5 and $1.5 \mathrm{~Hz}, \mathrm{H}-6$ ), 8.04 (dd, $1 \mathrm{H}, J 7.5$ and $1.5 \mathrm{~Hz}, \mathrm{H}-8), 8.10$ (dd, $1 \mathrm{H}, J 7.5$ and $1.5 \mathrm{~Hz}$, $\mathrm{H}-5) ;{ }^{13} \mathrm{C}$ NMR (125.0 MHz, DMSO- $\left.d_{6}\right) \delta 15.4$ (C-5'), 20.3 (C-4'), $\left.22.1\left[\mathrm{C}\left(\mathrm{CH}_{3}\right)_{2}\right], 22.9\left(\mathrm{C}-1^{\prime}\right), 23.7\left[\mathrm{C}^{\left(\mathrm{CH}_{3}\right.}\right)_{2}\right]$, 44.6 (C-5"), $51.9\left(\mathrm{OCH}_{3}\right), 78.9$ (C-2"), 82.1 (C-3"), 82.5 (C-4"), 106.4 (C-1"), 109.1 [C( $\left.\left.\mathrm{CH}_{3}\right)_{2}\right], 112.9$ (C-3), 119.9 (C-3'), 123.0 (C-5) or (C-8), 123.2 (C-5) or (C-8), 127.7 (C-4a), 129.3 (C-8a), 130.0 (C-2'), 132.0 (C-6), 143.4 (C-
2), 179.1 (C-4), 180.0 (C-1); HRMS (ESI) [M+Na] ${ }^{+}$Found: 450.1915. Calc. for $\mathrm{C}_{24} \mathrm{H}_{29} \mathrm{NO}_{6} \mathrm{Na}$ : 450.1818 .

2-(6"-Deoxy-1",2":3",4"-di-O-isopropylidene-D-galactopyranos-6"'-yl)-amino-3-(3'-methyl-buten-2'-yl)-1,4naphthoquinone, $\mathbf{7 b}$

The reaction of naphthoquinone $\mathbf{8 b}$ (158 $\mathrm{mg}, 1 \mathrm{mmol})$ and aminocarbohydrate $\mathbf{9 b}(128 \mathrm{mg}, 0.5 \mathrm{mmol})$ formed $\mathbf{7 b}$ (77 $\mathrm{mg}, 0.16 \mathrm{mmol}, 32 \%$ ) as brown oil; IR $v_{\max } / \mathrm{cm}^{-1} 1600$ and $1668(\mathrm{C}=\mathrm{O}), 1570(\mathrm{C}=\mathrm{C}), 3347(\mathrm{~N}-\mathrm{H}) ;{ }^{1} \mathrm{H}$ NMR $(500$ $\left.\mathrm{MHz}, \mathrm{DMSO}-d_{6}\right) \delta 1.21$ (s, 3H, $\left.\mathrm{C}\left(\mathrm{CH}_{3}\right)_{2}\right), 1.23$ (s, 3H, $\left.\mathrm{C}\left(\mathrm{CH}_{3}\right)_{2}\right), 1.30\left(\mathrm{~s}, 3 \mathrm{H}, \mathrm{C}\left(\mathrm{CH}_{3}\right)_{2}\right), 1.34$ (s, 3H, $\left.\mathrm{C}\left(\mathrm{CH}_{3}\right)_{2}\right), 1.62$ (H-1'), 1.69 (H-5'), 3.27 (dd, 1H, J 7.0 Hz, H-4'), 3.60 (dd, $1 \mathrm{H}, J 14.0$ and $8.0 \mathrm{~Hz}, \mathrm{H}-6$ "), 3.72 (dd, $1 \mathrm{H}, J 14.0$ and 4.0 Hz, H-6"'), 3.84-3.85 (m, 1H, H-5"), 4.19 (dd, 1H, J 8.0 and $1.5 \mathrm{~Hz}, \mathrm{H}-4$ "), 4.30 (dd, $1 \mathrm{H}, J 4.5$ and $2.5 \mathrm{~Hz}, \mathrm{H}-2$ "), 4.58 (dd, $1 \mathrm{H}, J 8.0$ and $2.5 \mathrm{~Hz}, \mathrm{H}-3$ "), $5.02-5.04(\mathrm{~m}, 1 \mathrm{H}$, H-3'), 5.42 (d, 1H, J 5.0 Hz, H-1"), 6.36 (dd, 1H, J 6.5 and $6.0 \mathrm{~Hz}, \mathrm{~N}-\underline{\mathrm{H}}), 7.69$ (td, $1 \mathrm{H}, J 7.7$ and $1.5 \mathrm{~Hz}, \mathrm{H}-7), 7.77$ (td, $1 \mathrm{H}, J 7.5$ and $1.5 \mathrm{~Hz}, \mathrm{H}-6), 7.90(\mathrm{dd}, 1 \mathrm{H}, J 7.8$ and 1.5 $\mathrm{Hz}, \mathrm{H}-8), 7.91$ (dd, $1 \mathrm{H}, J 7.5$ and $1.5 \mathrm{~Hz}, \mathrm{H}-5$ ); ${ }^{13} \mathrm{C}$ NMR $\left(125.0 \mathrm{MHz}, \mathrm{DMSO}-d_{6}\right) \delta 18.20$ (C-5'), 23.0 (C-4'), 24.4 $\left[\mathrm{C}\left(\mathrm{CH}_{3}\right)_{2}\right], 25.0\left[\mathrm{C}\left(\mathrm{CH}_{3}\right)_{2}\right], 25.6\left(\mathrm{C}-1^{\prime}\right), 25.8\left[\mathrm{C}^{2}\left(\mathrm{CH}_{3}\right)_{2}\right]$, $26.1\left[\mathrm{C}\left(\mathrm{CH}_{3}\right)_{2}\right], 44.9$ (C-6" and C-6"'), 66.9 (C-5"), 70.1 (C-2"), 70.5 (C-3"), 71.0 (C-4"), 95.9 (C-1"), 108.2

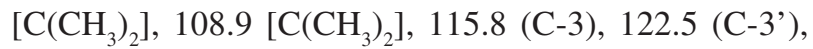
125.6 (C-8), 125.9 (C-5), 130.6 (C-4a), 131.5 (C-8a), 132.7 (C-7), 134.7 (C-6), 146.9 (C-2), 181.8 (C-4), 182.8 (C-1).

2-(5'"'-Deoxy-1"',2','-O-isopropylidene-D-xylofuranos5'"yl)-amino-3-(3'-methyl-buten-2'-yl)-1,4- naphthoquinone, $7 c$

The reaction of naphthoquinone $8 \mathbf{b}$ (158 $\mathrm{mg}, 1 \mathrm{mmol}$ ) of aminocarbohydrate $9 \mathbf{c}(95 \mathrm{mg}, 0.5 \mathrm{mmol})$ led to formation of 7c (74 mg, $0.18 \mathrm{mmol}, 37 \%)$ as a brown oil; IR $v_{\text {max }}$ ' $\mathrm{cm}^{-1} 1600$ and $1670(\mathrm{C}=\mathrm{O}), 1600(\mathrm{C}=\mathrm{C}), 3343(\mathrm{~N}-\mathrm{H})$, 3400-3500 (OH); ${ }^{1} \mathrm{H}$ NMR (500 MHz, DMSO- $\left.d_{6}\right) \delta 1.33$ (s, 3H, $\left.\mathrm{C}\left(\mathrm{CH}_{3}\right)_{2}\right), 1.47$ (s, 3H, $\left.\mathrm{C}\left(\mathrm{CH}_{3}\right)_{2}\right), 1.75$ (H-1'), 1.82 (H-5'), 3.38 (dd, 1H, J 15.5 and $6.0 \mathrm{~Hz}, \mathrm{H}-4$ '), 3.43 (dd, $1 \mathrm{H}, J 15.5$ and $6.5 \mathrm{~Hz}, \mathrm{H}-4$ "), 3.82-3.90 (m, 2H, H-5" " and H-5"'”), 4.11 (d, 1H, J 3.0 Hz, H-3"'), 4.31 (td, 1H, J 6.0 and $3.0 \mathrm{~Hz}, \mathrm{H}-4$ "'), 4.54 (d, 1H, J 3.5 Hz, H-2"'), 5.13 (dd, $1 \mathrm{H}, J 6.5$ and $\left.6.0 \mathrm{~Hz}, \mathrm{H}-3^{\prime}\right), 5.58(\mathrm{~d}, 1 \mathrm{H}, J 5.0 \mathrm{~Hz}, \mathrm{OH}$ ), 5.96 (d, 1H, $J 3.5 \mathrm{~Hz}, \mathrm{H}-1$ '”), 6.53 (dd, 1H, $J 6.5$ and 6.0 $\mathrm{Hz}, \mathrm{N}-\mathrm{H}), 7.81$ (td, $1 \mathrm{H}, J 7.5$ and $1.5 \mathrm{~Hz}, \mathrm{H}-7), 7.90$ (td, $1 \mathrm{H}, J 7.5$ and $1.5 \mathrm{~Hz}, \mathrm{H}-6), 8.04$ (dd, $1 \mathrm{H}, J 7.5$ and 1.5 $\mathrm{Hz}, \mathrm{H}-8), 8.05$ (dd, $1 \mathrm{H}, J 7.5$ and $1.5 \mathrm{~Hz}, \mathrm{H}-5) ;{ }^{13} \mathrm{C}$ NMR (125.0 MHz, DMSO- $d_{6}$ ) $\delta 15.4$ (C-5'), 20.3 (C-4'), 22.2 (C-1'), $22.9\left[\mathrm{C}\left(\mathrm{CH}_{3}\right)_{2}\right], 23.7\left[\mathrm{C}\left(\mathrm{CH}_{3}\right)_{2}\right], 43.2\left(\mathrm{C}-5{ }^{\prime \prime}\right.$ ' and C-5"'"), 78.9 (C-3"'), 82.1(C-4"'), 82.5 (C-2"'), 106.4

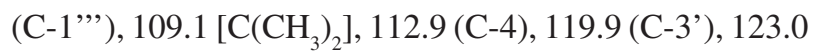


(C-8), 123.2 (C-5), 129.3 (C-4a), 129.8 (C-7), 130.0 (C-8a), 132.1 (C-6), 143.4 (C-2), 181.9 (C-4), 183.0 (C-1); HRMS (ESI) $\left[\mathrm{M}^{+} \mathrm{Na}\right]^{+}$Found: 436.1750. Calc. for $\mathrm{C}_{23} \mathrm{H}_{27} \mathrm{NO}_{6} \mathrm{Na}$ : 436.1730 .

2-(2'-Deoxy-D-glucopyranos-2'-yl)-amino-1,4- naphthoquinone, $6 \mathrm{~d}$

The reaction of naphthoquinone $7 \mathbf{a}(316 \mathrm{mg}, 2 \mathrm{mmol})$ and aminocarbohydrate $\mathbf{8 d}$ (384 $\mathrm{mg}, 1 \mathrm{mmol})$ led to formation of 2-(2'-deoxy-D-glucopyranos-2'-yl) amino1,4-naphthoquinone $\mathbf{6 d}$, as mixture of two diastereoisomers, $\alpha: \beta(1: 1)$, in $40 \%$ yield.

\section{2-(2'-Deoxy-1,3,4,6-tetra-O-acetyl- $\alpha$-D-glucopyranos-2'-} yl)-amino-1,4- naphthoquinone, $\boldsymbol{\sigma} \boldsymbol{e}$

Cold acetic anhydride $(3.0 \mathrm{~mL})$ was added to a stirred suspension of brown oil $\mathbf{6 d}(1,120 \mathrm{mg}, 3.36 \mathrm{mmol})$ in anhydrous pyridine $(5.4 \mathrm{~mL})$ at $0{ }^{\circ} \mathrm{C}$. The mixture was stirred at this temperature until dissolution was complete; it was then kept at room temperature for $12 \mathrm{~h}$. The solution was poured into cold water $(15 \mathrm{~mL})$ and cooled for $1 \mathrm{~h}$. The resulting solid was separated by filtration, washed with water, and dried over phosphorous pentoxide. The product was chromatographed on a silica gel column, using $50 \%$ $n$-hexane/ethyl acetate as mixture eluent, giving a brown solid in $60 \%$ yield as a mixture of anomers (3:1), 6e being the predominant isomer. IR $v_{\max } / \mathrm{cm}^{-1} 1610(\mathrm{C}=\mathrm{O}), 1677$ $(\mathrm{C}=\mathrm{O}), 1750(\mathrm{C}=\mathrm{O}), 1576(\mathrm{C}=\mathrm{C}), 1221(\mathrm{C}-\mathrm{O}), 3365$ $(\mathrm{N}-\mathrm{H}) ;{ }^{1} \mathrm{H}$ NMR $\left(500 \mathrm{MHz}, \mathrm{DMSO}-d_{6}\right) \delta 1.37(\mathrm{~s}, 3 \mathrm{H}$, $\left.\mathrm{CH}_{3} \mathrm{C}=\mathrm{O}\right), 1.48$ (s, 3H, $\left.\mathrm{CH}_{3} \mathrm{C}=\mathrm{O}\right), 1.77$ (s, 3H, $\left.\mathrm{CH}_{3} \mathrm{C}=\mathrm{O}\right)$, 1.84 (s, 3H, $\left.\mathrm{CH}_{3} \mathrm{C}=\mathrm{O}\right)$; 4.13-4.17 (m, 1H, H-5'), 4.26-4.30 (m, 1H, H-6' and H-6"), 4.33 (dd, 1H, J 12.5 and $4.0 \mathrm{~Hz}$, H-6"), 4.52 (td, $1 \mathrm{H}, J 10.0$ and $3.5 \mathrm{~Hz}, \mathrm{H}-2$ '), 5.29 (dd, $1 \mathrm{H}, J 9.5$ and $\left.9.5 \mathrm{~Hz}, \mathrm{H}-4^{\prime}\right), 5.53$ (dd, $1 \mathrm{H}, J 10.0$ and 9.5 Hz, H-3'), 6.23 (d, 1H, J 4.0 Hz, H-1'), 6.33 (s, 1H, H-3), $6.56(\mathrm{~d}, 1 \mathrm{H}, J 9.5 \mathrm{~Hz}, \mathrm{~N}-\mathrm{H}), 7.87 \mathrm{td}, 1 \mathrm{H}, J 7.5$ and $1.5 \mathrm{~Hz}$, H-7), 7.96 (td, $1 \mathrm{H}, J 7.5$ and $1.5 \mathrm{~Hz}, \mathrm{H}-6), 8.06$ (dd, 1H, $J$ 7.5 and $1.5 \mathrm{~Hz}, \mathrm{H}-8), 8.09$ (dd, $1 \mathrm{H}, J 7.5$ and $1.5 \mathrm{~Hz}, \mathrm{H}-5)$; ${ }^{13} \mathrm{C}$ NMR $\left(125.0 \mathrm{MHz}, \mathrm{DMSO}-d_{6}\right) \delta 20.0\left(\mathrm{CH}_{3} \mathrm{C}=\mathrm{O}\right), 20.5$ $\left(\mathrm{CH}_{3} \mathrm{C}=\mathrm{O}\right), 20.6\left(\mathrm{CH}_{3} \mathrm{C}=\mathrm{O}\right), 20.7\left(\mathrm{CH}_{3} \mathrm{C}=\mathrm{O}\right), 52.7\left(\mathrm{C}-2{ }^{\prime}\right)$, 61.5 (C-6' and C-6’), 67.7 (C-4'), 69.4 (C-5'), 71.2 (C-3'), 89.1 (C-1'), 102.2 (C-3), 125.6 (C-8), 126.2 (C-5), 130.3 (C-8a), 132.7 (C-4a), 132.9 (C-7), 135.2 (C-6), 148.5 (C-2), $169.3\left(\mathrm{CH}_{3} \mathrm{C}=\mathrm{O}\right), 169.5\left(\mathrm{CH}_{3} \mathrm{C}=\mathrm{O}\right), 170.3\left(\mathrm{CH}_{3} \mathrm{C}=\mathrm{O}\right), 170.5$ $\left(\mathrm{CH}_{3} \mathrm{C}=\mathrm{O}\right), 181.3(\mathrm{C}-1), 182.6(\mathrm{C}-4)$; $\mathrm{HRMS}(\mathrm{ESI})\left[\mathrm{M}^{+} \mathrm{Na}\right]^{+}$ Found: 526.1317. Calcd for $\mathrm{C}_{24} \mathrm{H}_{25} \mathrm{NO}_{11} \mathrm{Na}$ : 526.1319.

\section{Conclusions}

We have developed a method for the synthesis of new series of substituted-2-aminonaphthoquinones 6a-d and $7 \mathbf{a}-\mathbf{c}$ by the reaction of 1,4-naphtoquinone (8a) or 2-methoxylapachol (8b) with different aminocarbohydrates 9a-d, under ultrasonic irradiation, which accelerate the 1,4-addition, in the absence of iodine (method B) being a faster and cleaner direct access for the synthesis of new sugar-based quinone compounds. Further efforts are being dedicated towards the evaluation of the biological profiles of this novel type of 1,4-naphthoquinone derivatives.

\section{Acknowledgments}

This work was supported by the Brazilian agency FAPERJ-PRONEX. Fellowship granted to UFF, by FAPERJ (E-26/171.512/2006) is gratefully acknowledged. We would like to thank the Universidade Federal do Rio de Janeiro, Departamento de Química Orgânica, LABEM Laboratório Multiusuário de Espectrometria de Massas for the mass spectra.

\section{References}

1. Mahapatra, A.; Mativandlela, S. P. N.; Binneman, B.; Fourie, P. B.; Hamilton, C. J.; Meyer, J. J. M.; van der Kooy, F.; Houghton, P.; Lall, N.; Bioorg. Med. Chem. 2007, 15, 7638.

2. Da Silva, M. N.; Ferreira, V. F.; De Souza, M. C. B. V.; Quim. Nova 2003, 26, 407.

3. Suh, M.-E.; Parka, S.-Y.; Lee, C.-O.; Bioorg. Med. Chem. 2001, 9, 2979; Da Silva, F. C.; Ferreira, S. B.; Kaiser, C. R.; Pinto, A. C.; Ferreira, V. F.; J. Braz. Chem. Soc. 2009, 20, 1478.

4. Francisco, A. I.; Casellato, A.; Neves, A. P.; Carneiro, J. W. M.; Vargas, M. D.; Visentin, L. C.; Magalhães, A.; Câmara, C. A.; Pessoa, C.; Costa-Lotufo, L. V.; Filho, J. D. B. M.; Moraes, M. O.; J. Braz. Chem. Soc. 2010, 21, 169.

5. Boudalis, A. K.; Policand, X.; Sournia-Saquet, A.; Donnadieu, B.; Tuchagues, J.-P.; Inorg. Chim. Acta 2008, 361, 1681.

6. Tonholo, J.; Freitas, L. R.; De Abreu, F. C.; Azevedo, D. C.; Zani, C. L.; De Oliveira, A. B.; Goulart, M. O. F.; J. Braz. Chem. Soc. 1998, 9, 163.

7. Da Silva, M. N.; Ferreira, S. B.; Jorqueira, A.; De Souza, M. C. B. V.; Pinto, A. V.; Kaiser, C. R.; Ferreira, V. F.; Tetrahedron Lett. 2007, 48, 6171.

8. De Moura, K. C. G.; Emery, F. S.; Neves-Pinto, C.; Pinto, M. C. F. R.; Dantas, A. P.; Salomão, K.; De Castro, S. L.; Pinto, A. V.; J. Braz. Chem. Soc. 2001, 12, 325.

9. Tandon, V. K.; Maurya, H. K.; Mishra, N. N.; Shukla, P. K.; Eur. J. Med. Chem. 2009, 44, 3130.

10. Camara, C. A.; Pinto, A. C.; Rosa, M. A.; Vargas, M. D.; Tetrahedron 2001, 57, 9569.

11. Asche, C.; Mini-Rev. Med. Chem. 2005, 5, 449.

12. Júnior, E. N. S.; Souza, M. C. B. V.; Pinto, A. V.; Pinto, M. C. F. R.; Goulart, M. O. F.; Barros, F. W. A.; Pessoa, C.; Costa- 
Lotufo, L. V.; Montenegro, R. C.; Moraes, M. O.; Ferreira, V. F.; Bioorg. Med. Chem. 2007, 15, 7035; Neves, A. P.; Barbosa, C. C.; Greco, S. J.; Vargas, M. D.; Visentin, L. C.; Pinheiro, C. B.; Mangrich, A. S.; Barbosa, J. P.; da Costa, G. L.; J. Braz. Chem. Soc. 2009, 20, 712.

13. Kapadia, G. J.; Azuine, M. A.; Balasubramanian, V.; Sridhar, R.; Pharmacol. Res. 2001, 43, 363.

14. Yuk, D.-Y.; Ryu, C.-K.; Hong, J.-T.; Chung, K.-H.; Kang, W.-S.; Kim, Y.; Yoo, H.-S.; Lee, M.-K.; Lee, C.-K.; Yun, Y.-P.; Biochem. Pharmacol. 2000, 60, 1001.

15. Silva, T. M. S.; Camara, C. A.; Barbosa, T. P.; Soares, A. Z.; Cunha, L. C.; Pinto, A. C.; Vargas, M. D.; Bioorg. Med. Chem. 2005, 13, 193.

16. Oliveira, C. G. T.; Miranda, F. F.; Ferreira, V. F.; Freitas, C. C.; Rabello, R. F.; Carballido, J. M.; Corrêa, L. C. D.; J. Braz. Chem. Soc. 2001, 12, 339.

17. Fieser, L. F.; Hartwell, J. L.; J. Am. Chem. Soc. 1935, 57, 1482.

18. Couladouros, E. A.; Plyta, Z. F.; Haroutounian, S. A.; J. Org. Chem. 1997, 62, 6.

19. Cunha, A. S.; Lima, E. L. S.; Pinto, A. C.; Esteves-Souza, A.; Echevarria, A.; Câmara, C. A.; Vargas, M. D.; Torres, J. C.; J. Braz. Chem. Soc. 2006, 17, 439.

20. Francisco, A. I.; Vargas, M. D.; Carneiro, J. W. M.; Lanznaster, M.; Torres, J. C.; Câmara, C. A.; Pinto, A. C.; J. Mol. Struct. 2008, 891, 228.

21. Win, T.; Bittner, S.; Tetrahedron Lett. 2005, 46, 3229.

22. Illos, R. A.; Harlev, E.; Bittner; S.; Tetrahedron Lett. 2005, 46, 8427.

23. Tapia, R. A.; Cantuarias, L.; Cuéllar, M.; Villena, J.; J. Braz. Chem. Soc. 2009, 20, 999.

24. Valderrama, J. A.; Ibacache, J. A.; Arancibia, V.; Rodriguez, J.; Theoduloz, C.; Bioorg. Med. Chem. 2009, 17, 2894.

25. Tandon, V. K.; Maurya, H. K.; Tetrahedron Lett. 2009, 50, 5896.

26. Couladouros, E. A.; Plyta, Z. F.; Papageorgiou, V. P.; J. Org. Chem. 1996, 61, 3031.

27. Liu, B.; Ji, S.-J.; Synth. Commun. 2008, 38, 1201.

28. Niedermeyer, T. H. J.; Lalk, M.; J. Mol. Catal. B: Enzym. 2007, $45,113$.

29. Bittner, S.; Meenakshi, C.; Temtsin, G.; Tetrahedron 2001, 57, 7423.

30. Messori, L.; Temperini, C.; Piccioli, F.; Animati, F.; Di Bugn, C.; Orioli, P.; Bioorg. Med. Chem. 2001, 9, 1815.

31. Hauser, F. M.; Hu; X.; Org. Lett. 2002, 4, 977.
32. Molina-Ruiz, R.; Saíz-Urra, L.; Rodríguez-Borges, J. E.; PérezCastillo, Y.; González, M. P.; García-Mera, X.; Cordeiro, M. N. D. S.; Bioorg. Med. Chem. 2009, 17, 537.

33. Pereira, L. O. R.; Cunha, A. C.; Souza, M. C. B. V.; Ferreira, V. F.; J. Braz. Chem. Soc. 2002, 13, 368.

34. Santos, F. C.; Abreu, P.; Castro, H. C.; Paixão, I. C. P. P.; Cirne-Santos, C. C.; Giongo, V.; Barbosa, J. E.; Simonetti, B. R.; Garrido, V.; Bou-Habib, D. C.; Silva, D. O.; Batalha, P. N.; Temerozo, J. R.; De Souza, T. M.; Nogueira, C. M.; Cunha, A. C.; Rodrigues, C. R.; Ferreira, V. F.; Souza, M. C. B. V.; Bioorg. Med. Chem. 2009, 17, 5476.

35. Secrist, J. A.; Logue, M. W.; J. Org. Chem. 1972, 37, 335.

36. Sharma, G. V. M.; Gopinath, T.; Tetrahedron Lett. 2001, 42, 6183.

37. Yang, J.; Fu, X.; Jia, Q.; Shen, J.; Biggins, J. B.; Jiang, J.; Zhao, J.; Schmidt, J. J.; Wang, P. G.; Thorson, J. S.; Org. Lett. 2003, 5, 2223.

38. Roslund, M. U.; Klika, K. D.; Lettila, R. L.; Tahtinen, P.; Sillanpaa, R.; Leino, R.; J. Org. Chem. 2004, 69, 18.

39. Liberek, B.; Melcer, A.; Osuch, A.; Wakiec, R. ; Milewskib, S.; Wisniewskia, A.; Carbohydr. Res. 2005, 340, 1876.

40. Medgyes, A.; Farkas, E.; Lipták, A.; Pozsgay, V.; Tetrahedron 1997, 53, 4159.

41. Arakawa, K.; Miyasaka, T.; Hamamichi, N.; Chem. Lett. 1976, 1119.

42. Crystallographic data (excluding structure factors) for the structures in this paper have been deposited in the Cambridge Crystallographic Data Centre as supplementary publication number CCDC 762331. Copies of the data can be obtained, free of charge, via www.ccdc.cam.ac.uk/conts/retrieving.html or from the Cambridge Crystallographic Data Centre, CCDC, 12 Union Road, Cambridge CB2 1EZ, UK; fax: +44 1223336033. E-mail: deposit@ccdc.cam.ac.uk.

43. Horton, D.; Levine, B. R.; Norris, P.; Luck, R. L.; Silverton, J. V.; Acta Crystallogr., Sect. C: Cryst. Struct. Commun. 1997, 53,120 .

44. Mason, T. J.; Chem. Soc. Rev. 1997, 26, 443; Guo, W.-X.; Jin, H.-L.; Chen, J.-X.; Chen, F.; Ding, J.-C.; Wu, H.-Y.; J. Braz. Chem. Soc. 2009, 20, 1674.

Submitted: March 3, 2010 Published online: August 24, 2010 